\title{
Assessment of Electrical Influence of Multiple Piezoelectric Transducers' Connection on Actual Satellite Vibration Suppression
}

\author{
Shigeru Shimose, ${ }^{1}$ Kanjuro Makihara, ${ }^{2}$ Kenji Minesugi, ${ }^{1}$ and Junjiro Onoda ${ }^{1}$ \\ ${ }^{1}$ Institute of Space and Astronautical Science (ISAS), Japan Aerospace Exploration Agency (JAXA), 3-1-1 Yoshinodai, \\ Chuo-Ward, Sagamihara, Kanagawa, 252-5210, Japan \\ ${ }^{2}$ Department of Aerospace Engineering, Tohoku University, 6-6-01 Aoba, Aramaki, Aoba-Ward, Sendai 980-8579, Japan
}

Correspondence should be addressed to Shigeru Shimose, shimose.shigeru@jaxa.jp

Received 6 September 2010; Accepted 9 March 2011

Academic Editor: Osama J. Aldraihem

Copyright ( $) 2011$ Shigeru Shimose et al. This is an open access article distributed under the Creative Commons Attribution License, which permits unrestricted use, distribution, and reproduction in any medium, provided the original work is properly cited.

\begin{abstract}
We conduct comprehensive investigation of a semiactive vibration suppression method using piezoelectric transducers attached to structures. In our system, piezoelectric transducers are connected to an electric circuit composed of the diodes, an inductance, and a selective switch. Our method (SSDI) makes better use of counterelectromotive force to suppress the vibration, instead of simple dissipation of vibration energy. We use an actual artificial satellite to verify their high performance compared to conventional semi-active methods. As a consequence, we demonstrate that our semi-active switching method can suppress the vibration of the real artificial satellite to as much as $50 \%$ amplitude reduction. In our experiment, we reveal that the suppression performance depends on how multiple piezoelectric transducers are connected, namely, their series or parallel connection. We draw two major conclusions from theoretical analysis and experiment, for constructing effective semi-active controller using piezoelectric transducers. This paper clearly proves that the performance of the method is the connection (series or parallel) of multiple piezoelectric transducers and the their resistances dependent on frequency.
\end{abstract}

\section{Introduction}

Space structures, such as the space station or artificial satellites, are built for the purpose of highly demanding space missions. Large solar paddles and bulky antennas are necessary for the space missions. However, since the launch ability of rockets strictly limits the payload weight, space structures have to extremely minimize their weight, which results in the use of flexible structures and spindly members. Therefore, they are more prone to vibrations compared with structures on earth with less weight restriction. Moreover, space structures and artificial satellites are exposed to severe vibration environment at the launch stage. The relaxation of such a severe condition is an important issue in order to improve their reliability and to reduce their development costs. Many methods of vibration suppression have been studied and proposed [1-6].
There is currently a large effort underway to effectively suppress the vibration of structures. Vibration control methods are roughly categorized into two groups, that is, active and passive methods [1-6]. Active vibration control methods usually have high performance in vibration suppression [13]. In the space field, the active rack isolation system (ARIS) [1] of the International Space Station is famous as the active vibration control system composed of eight voice-coil actuators in order to maintain microgravity environment. Generally, constructing accurate mathematical model and tuning controller's parameters are indispensable to make active vibration controls feasible. However, it is usually quite difficult both to construct precise mathematical models and to tune parameters accurately. Active vibration control systems may become unstable if the control is improperly designed. Active vibration control methods need external energy supply to the system to suppress vibration. It is their 
major drawback from the viewpoint of the restriction of power resource of isolated satellites in space.

On the other hand, passive vibration control methods use energy dissipative mechanism by using dampers, frictional devices, electrical resistors, and so on [4-6]. In the space field, the Hubble Space Telescope (HST) is probably the most known example of a spacecraft that includes a passive damper vibration suppression system [4]. Since passive approaches do not supply energy to the system, passive systems are always stable. Passive methods are easier to be implemented in actual systems than complicated active methods, because they do not need controllers, sensors, or filters. However, in most cases, passive systems do not provide satisfactory performance in vibration suppression.

We had sought a new approach to solve the dilemma between active and passive vibration controls in robustness and suppression performance. A semiactive approach is one of practical answers both to reduce disadvantages of active systems and to maintain advantages of passive systems. So far, many studies of semiactive vibration suppression methods have been reported, and they often used piezoelectric actuators for vibration suppression [7-14]. Some researchers have studied a semiactive approach to suppress vibration by using piezoelectric actuators with switchable circuits [914]. Clark [9] proposed a switchable stiffness element composed of a piezoelectric actuator and a shunt circuit (State switching). Richard et al. [10] proposed to suppress vibration by switching a resistive shunt circuit in synchronization with structural vibration (known as SSD). Richard et al. [11], Corr and Clark [12], and Onoda et al. [13] proposed to use an inductive circuit. Depending on their circuit components and their switching strategies, this approach is called SSDI, LR-switching, RL-shunt, and so on. By shunting the transducer in short time, the counterelectromotive force generated by the inductor and the restored electric charge due to the piezoelectric effect can reserve the polarity of the charge stored in it. Accordingly, the stored electrical energy in the transducer is recycled rather than immediately dissipated, which is called an energy-recycling semiactive mechanism. Especially, Onoda et al. [13] showed that it is possible both to select and to suppress arbitrary vibration modes among all multiple modes. Makihara et al. [14] proposed a novel selfsensing technique using one piezoelectric transducer as both an actuator and a sensor. We expect this promising energyrecycling concept to be used in many applications. Qui et al. [15] recently presented an extensive overview of switching controls using piezoelectric materials, which provides the history and importance of this research field.

\section{Problem Statements and Research Objectives}

The final goal of our study is to apply the energy-recycling semiactive vibration suppression method to actual artificial satellites. Now, that we apply our method to the real-size satellite, many piezoelectric transducers are needed. The simplest way, all piezoelectric transducers are connected to their electric circuits, and these all are controlled independently. In this idea is that damping capability rises up with the number of piezoelectric transducers. Since the weight can be heavy due to many numbers of electric circuits and controllers, the simplest idea is not suitable to space structures. More sophisticated idea is that multiple piezoelectric transducers are connected in series and parallel as one group that connects each own electric circuit and controller. We focus on the study of relation between the damping capability and the electrical connections (series/parallel) of multiple piezoelectric transducers. These all are the major objectives of this paper.

Firstly, we will confirm the validity of our switching vibration suppression performance using a large-size actual satellite. Surprisingly, the acceleration reduces to as much as $50 \%$ of the original amplitude. This reduction number is quite striking to many researchers who devote themselves to space industries, as well as various engineering applications. This is because the validity experiment uses the actual artificial satellite, instead of easy-to-use experimental equipments for small laboratories.

Secondly, we will reveal theoretical analysis that a connection of the multiple piezoelectric transducers can exert an influence on the damping capability. We find a discrepancy between theoretical analysis result and experimental result. Furthermore, an intriguing discrepancy of damping capability between series/parallel connections will be clarified.

Thirdly, we will seek for the cause for the discrepancy that we encounter at theoretical speculation. In particular, we then focus on the internal resistance of piezoelectric transducers. The significance of the frequency-dependant internal resistances of piezoelectric transducers will be revealed by experimental data. The relationship between the internal resistance and electrical natural frequency will solve the cause of discrepancy issue that we come to grip with and will enrich the knowledge our understanding of the piezoelectric switching control. In this paper, by using actual artificial satellites (Figure 1), we investigate vibration suppression performance of the energy-recycling semiactive method. Figure 2 shows an artificial satellite (more specifically speaking, an interplanetary space probe "Planet-A") [16]. STM is a mechanical model for qualifying its structural design, which is completely the same as the actual artificial satellite in space. The vibration of the actual satellite is excited as a shaker shown in Figure 1. The shaker is hung from the crane and is connected to the pedestal of the high-gain antenna. In the actual condition of rocket liftoff, force excitation is input in the vertical direction through the downside connection. In our experimental implementation, the satellite is jolted on the base, and the excited force is applied in the vertical direction from the upper side. The attached piezoelectric transducers are the PZT C-91H type and are manufactured by Fuji Ceramic Co. Each has a length of $40 \mathrm{~mm}$, a width of $10 \mathrm{~mm}$, and a thickness of $0.5 \mathrm{~mm}$. 51 sheets of piezoelectric transducers are bonded to the both sides of the top panel that is composed of the honeycomb GFRP sheet sandwich. They are placed near the inside edge of the top panel, according to the preliminary FEM analysis to grasp their optimal position.

These researches are very important for optimizing the damping efficiency in SSDI by using multiple piezoelectric transducers. This paper demonstrates the relation between 


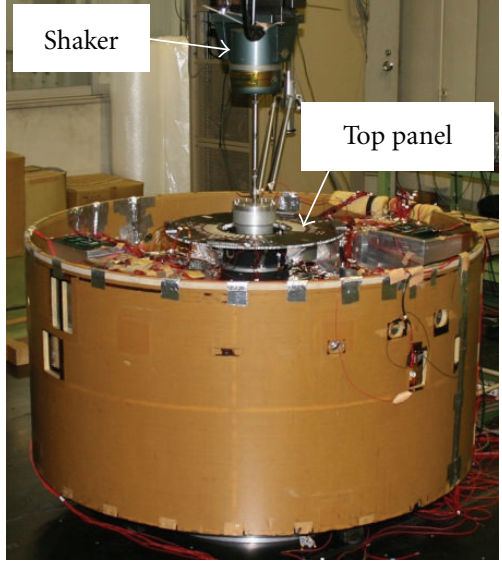

(a)

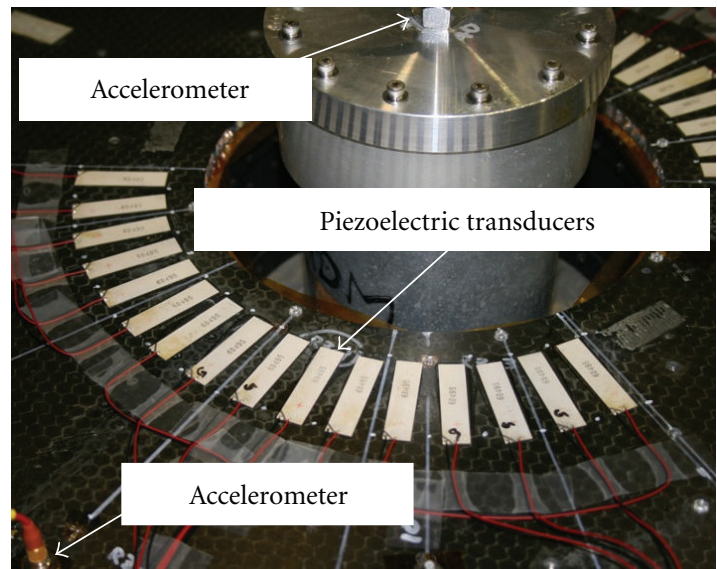

(b)

Figure 1: Photos of vibration suppression experiments of satellite "Planet-A" and bonded piezoelectric transducers.

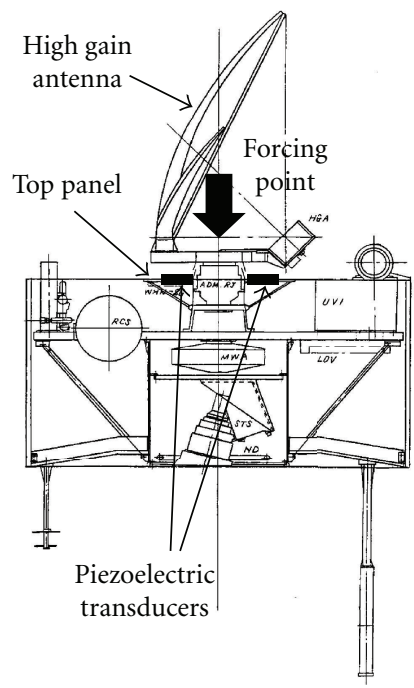

(a)

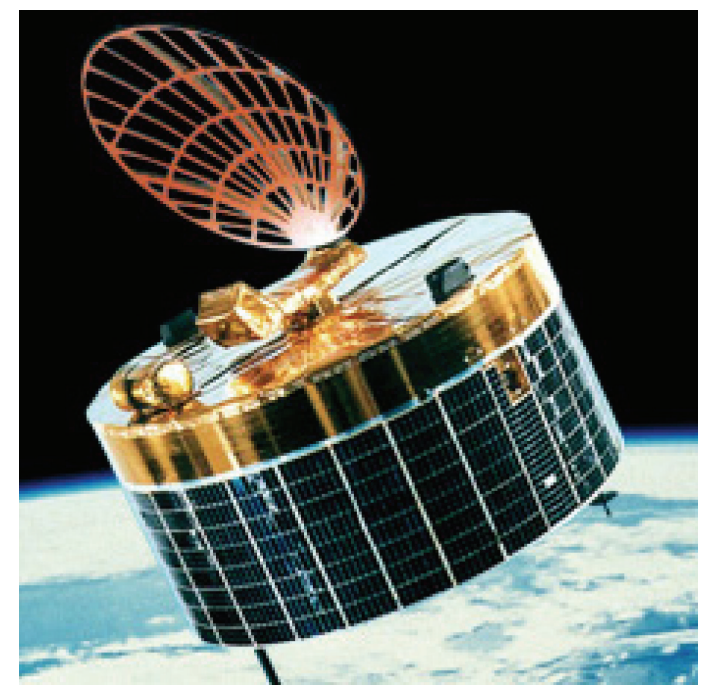

(b)

FIGURE 2: Cross-sectional view of satellite "Planet-A" and operational-concept rendering.

vibration suppression performance and piezoelectric transducer's connection (series/parallel). Theoretical analysis of energy-recycling semiactive vibration suppression using multiple piezoelectric transducers is presented. Next, it shows the details of the experiment using the actual artificial satellite, and the effective of our method. Lastly, theoretical results compared with experimental result. We clarify the cause of discrepancy between theoretical and experimental results.

\section{Theoretical Analysis}

3.1. Switching Method for Semi-Active Vibration Suppression. A basic idea of the energy-recycling semiactive mechanism is has been reported in [9-13]. However, for a better understanding, a brief explanation is presented here with a simple SDOF system shown in Figure 3. The system is composed of a mass, support structures, a piezoelectric transducer, and a switchable inductive circuit. The linear characteristic of the piezoelectric transducer [17] is written as

$$
f=k_{a} x-b_{a} Q, \quad V=-b_{a} x+\frac{Q}{C_{a}^{S}},
$$

where $f$ is the applied force, $x$ is the elongation of the piezoelectric transducer, $Q$ is the electric charge stored in the transducer, and $V$ is the piezoelectric voltage across two electric terminals. $C_{a}^{S}$ is the constant-elongation piezoelectric capacitance, and $b_{a}$ is the piezoelectric constant. The piezoelectric transducer is modeled with a voltage generator $V_{a}$ and a capacitor $C_{a}^{S}$. Here, let us assume that the SDOF system that has a natural frequency of $\omega$ is vibrating as

$$
x=a \sin (\omega t) .
$$


Active controls can freely change the value of $Q$ for vibration control. The optimal control charge (defined as $Q_{T}$ ) is derived from active control schemes. For instance, the direct velocity feedback control [18] stipulates its control input as

$$
Q_{T}=-F \frac{d x}{d t},
$$

where $F$ is the feedback gain.

In contrast to the above-mentioned active controls, our switching system (Figure 3 ) has only a selective switch to change its electrical state. This is the semiactive method that does not have an external power source and that cannot change the value of $Q$ as expected. Our switching logic is that the selective switch is connected to point 1 or 2 in order to make the polarity of $Q$ the same as that of $Q_{T}$. The switching logic [13] is written as follows:

when $Q_{T}<0$, turn the switch to point 1,

when $Q_{T} \geq 0$, turn the switch to point 2 .

We assume that $Q$ is initially zero at $t=0$. Equation (1) indicates that $V$ is negative when $x$ is positive and $Q$ is zero. While the switch is positioned on point 1 the diode at point 1 , prevents electric current from flowing in the circuit. Consequently, $x$ and $Q$ are zero, and $V$ is $-b_{a} a$ just before turning the switch at $t=\pi /(2 \omega)$. After the switch is connected to point 2 at $t=\pi /(2 \omega)$, electric current flows in the inductive circuit. The governing equation of the electric the circuit while the current flows is

$$
L \ddot{Q}+R \dot{Q}+\frac{Q}{C_{a}^{S}}=b_{a} x,
$$

which means that $\omega \sqrt{L C_{a}^{S}} \ll 1$ according to [13]. We also assume that electric current finishes flowing in a much shorter duration compared with the period of mechanical vibration. $x$ can, thus, be thought to remain $a$ during the current flow. The values of $Q$ and $V$ just after the current finishes flowing are

$$
Q=C_{a}^{S} b_{a} a\left(1+e^{-\zeta \pi}\right), \quad V=b_{a} a e^{-\zeta \pi}, \quad \zeta \equiv \frac{R}{2} \sqrt{\frac{C_{a}^{S}}{L}} .
$$

Despite $x$ and $V, Q$ remain constant, because diodes prevent current from flowing. At $t=(3 \pi) /(2 \omega), x$ change to $-a$ and $V$ becomes

$$
V=b_{a} a e^{-\zeta \pi}+2 b_{a} a .
$$

At this moment, the switch is turned to point 1 according to the control logic (equation (4)), and the current starts to flow. After the current flow stops due to the diode at point 1 , $Q$ and $V$ are

$$
\begin{gathered}
Q=-C_{a}^{S} b_{a} a\left(1+2 e^{-\zeta \pi}+e^{-2 \zeta \pi}\right), \\
V=-b_{a} a\left(2 e^{-\zeta \pi}+e^{-2 \zeta \pi}\right) .
\end{gathered}
$$

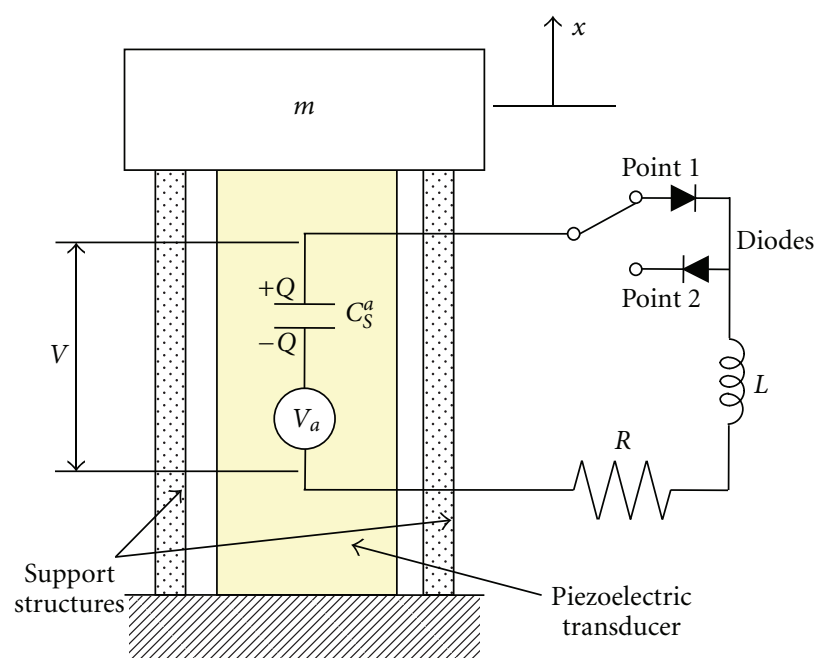

FIGURE 3: Simple SDOF system with a piezoelectric transducer and a switchable inductive circuit.

After many times of switching, the values of $Q$ and peak voltage converge to

$$
\begin{gathered}
|Q|=C_{a}^{S} b_{a} a \frac{1+\gamma}{1-\gamma}, \\
\left|V_{\text {Peak }}\right|=b_{a} a\left(\frac{1+\gamma}{1-\gamma}+1\right), \\
\gamma \equiv e^{-\zeta \pi} .
\end{gathered}
$$

Similarly, the dissipated energy $W_{D}$ at one cycle is

$$
W_{D}=4 C_{a}^{S} b_{a}^{2} a^{2} \frac{1+\gamma}{1-\gamma} .
$$

The parameter $\gamma$ is the direct relation to the dissipated energy and is called the voltage inversion coefficient [19]. It is defined as the ratio of voltages after and before voltage inversion. In the two following subsections, we will investigate damping capacity variation under two extreme assumptions of piezoelectric transducers' resistance. Consequently, we will draw very interesting conclusions from theoretical analyses under the two assumptions.

3.2. Dependence of Damping Capability on Multiple Transducers' Connection. We investigate the dependence of damping capability on how to connect $\mathrm{n}$ pieces of piezoelectric transducers. The damping capability is assessed in terms of the total energy dissipation per cycle of $\mathrm{n}$ transducers under the given control logic in (3) and (4). Importantly, in this subsection, we assume that the resistance of piezoelectric transducers is negligibly small compared with those of an inductor or electric harness. On one hand, the equation of the circuit in case of series connection is written as

$$
L \ddot{Q}+R \dot{Q}+n \frac{Q}{C_{a}^{S}}=n b_{a} x .
$$




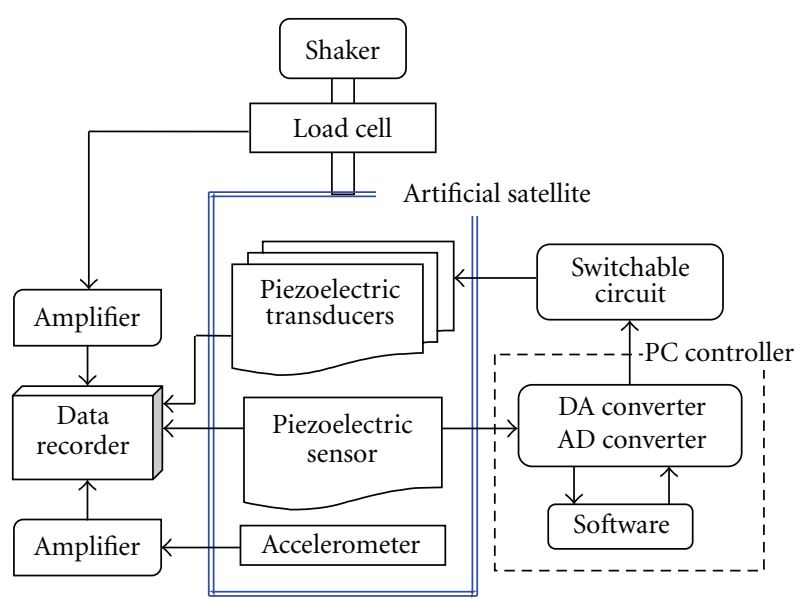

FIGURE 4: Schematic diagram of vibration suppression experiment.

On the other hand, in case of connection in parallel, the governing equation of the electric circuit is

$$
L \ddot{Q}+R \dot{Q}+\frac{Q}{n C_{a}^{S}}=b_{a} x,
$$

$R$ is mainly composed of an inductor and electric harness. By performing the same process to obtain (10), dissipated energies $W_{\mathrm{DS}}$ (Series connection) and $W_{\mathrm{DP}}$ (Parallel connection) at one cycle under steady-state condition converge to

$$
W_{\mathrm{DS}}=4 n C_{a}^{S} b_{a}^{2} a^{2} \frac{1+\gamma_{S}}{1-\gamma_{S}}, \quad W_{\mathrm{DP}}=4 n C_{a}^{S} b_{a}^{2} a^{2} \frac{1+\gamma_{P}}{1-\gamma_{P}},
$$

where

$$
\begin{array}{rlrl}
\gamma_{S} & \equiv e^{-\zeta_{S} \pi}, & \gamma_{P} & \equiv e^{-\zeta_{P} \pi}, \\
\zeta_{S} \equiv \frac{R}{2} \sqrt{\frac{C_{a}^{S}}{n L}}=\frac{\zeta}{\sqrt{n}}, & \zeta_{P} \equiv \frac{R}{2} \sqrt{\frac{n C_{a}^{S}}{L}}=\sqrt{n} \zeta .
\end{array}
$$

As long as $n \geq 2$, (14) derives an inequality

$$
\zeta_{S}<\zeta<\zeta_{P}
$$

From (14) and (15), we obtain

$$
\gamma_{P}<\gamma<\gamma_{S}
$$

Through (13)-(16), the relation of dissipated energies is written as

$$
W_{\mathrm{DP}}<W_{\mathrm{D}}<W_{\mathrm{DS}}
$$

From these theoretical analyses, we can conclude that the damping capability depends on the connection method of multiple piezoelectric transducers. Vibration suppression systems having series-connected piezoelectric transducers outperform ones having parallel-connected transducers. As the number of series-connected transducers increases, the damping capability becomes higher. That is a quite interesting and insightful conclusion; however, keep in mind that we assure that $R$ is mainly composed of an inductor and electrical harness.

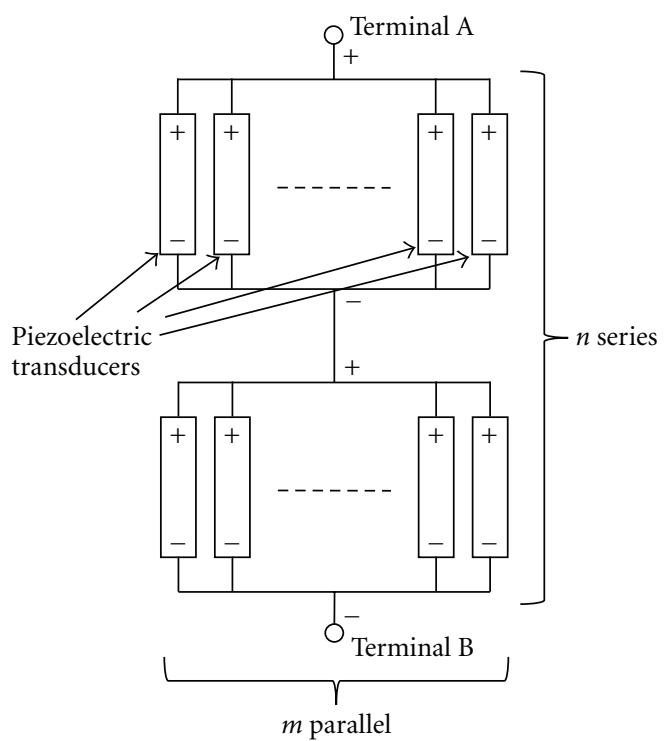

FIgURE 5: Connection of $m$ parallel and $n$ series of piezoelectric transducers in the circuit.

3.3. Dependence of Damping Capability on Resistance of Piezoelectric Transducers. In the previous subsection, the resistance of piezoelectric transducers is negligibly small. But actual piezoelectric transducers usually have large resistance value. In this subsection, we newly introduce the resistance of piezoelectric transducer, $R_{\text {pie }}$, and assume that it is large enough to neglect other components' resistance. The equation of the electric circuit in case of series connection is

$$
L \ddot{Q}+n R_{\mathrm{pie}} \dot{Q}+\frac{n Q}{C_{a}^{S}}=n b_{a} x .
$$

The equation of parallel connection is

$$
L \ddot{Q}+\frac{R_{\text {pie }}}{n} \dot{Q}+\frac{Q}{n C_{a}^{S}}=b_{a} x .
$$

Similarly to the previous procedures, dissipated energies $W_{\text {DSR }}$ (series connection with resistance consideration) and $W_{\text {DPR }}$ (parallel connection with Resistance consideration) at one cycle under steady-state condition converge to

$$
W_{\mathrm{DSR}}=4 n C_{a}^{S} b_{a}^{2} a^{2} \frac{1+\gamma_{\mathrm{SR}}}{1-\gamma_{\mathrm{SR}}}, \quad W_{\mathrm{DPR}}=4 n C_{a}^{S} b_{a}^{2} a^{2} \frac{1+\gamma_{\mathrm{PR}}}{1-\gamma_{\mathrm{PR}}},
$$

where

$$
\begin{gathered}
\gamma_{\mathrm{SR}} \equiv e^{-\zeta_{\mathrm{SR}} \pi}, \quad \gamma_{\mathrm{PR}} \equiv e^{-\zeta_{\mathrm{PR}} \pi}, \\
\zeta_{\mathrm{SR}} \equiv \frac{n R_{\mathrm{pie}}}{2} \sqrt{\frac{C_{a}^{S}}{n L}}=\frac{R_{\mathrm{pie}}}{2} \sqrt{\frac{n C_{a}^{S}}{L}}, \\
\zeta_{\mathrm{PR}} \equiv \frac{R_{\mathrm{pie}}}{2 n} \sqrt{\frac{n C_{a}^{S}}{L}}=\frac{R_{\mathrm{pie}}}{2} \sqrt{\frac{C_{a}^{S}}{n L}} .
\end{gathered}
$$

From (21), we obtain

$$
\zeta_{\mathrm{PR}}<\zeta<\zeta_{\mathrm{SR}}, \quad \gamma_{\mathrm{SR}}<\gamma<\gamma_{\mathrm{PR}}
$$




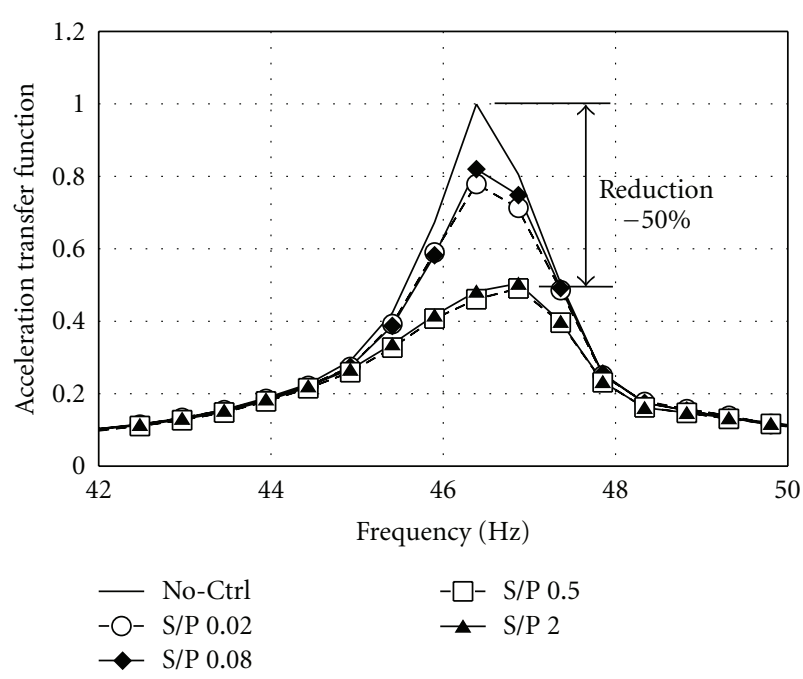

FIgURE 6: Damping capability of our switching method as a function of series/parallel ratio.

Accordingly the relation of these dissipated energies can newly be derived as

$$
W_{\mathrm{DSR}}<W_{\mathrm{D}}<W_{\mathrm{DPR}}
$$

It is concluded that the damping capability depends on the resistance of piezoelectric transducers. Surprisingly, comparing (17) and (23), we see a totally opposite inequity of the dissipated energies in light of parallel and series connection. Our two assumptions and consequent analyses lead an interesting problem presentation. We need to enrich our understanding of the relation between piezoelectric transducers' resistance and their electrical connection. To solve the essential discrepancy, in experiments using actual components, we will seek for the cause of the discrepancy.

\section{Experimental Analyses}

4.1. Experimental Configuration. The schematic diagram of our experiment is shown in Figure 4. One piezoelectric transducer is used as a piezoelectric sensor. The voltage value of the sensor is sent to the PC controller. The PC control software monitors, calculates, and determines the circuit switch-position according to both control logic and measured vibration data from the sensor. When the PC turns the switch position, the voltage's polarity of each piezoelectric transducer will be inversed.

4.2. Experimental Results. The connection of the transducers is composed of $m$ parallel and $n$ series, where $m$ and $n$ are integers (see Figure 5). It means that 50 transducers are divided into $n$ groups that are composed of $m$ transducers. $m$ transducers in each group are connected in parallel, and the $n$ groups are connected in series. $m \times n$ always equals 50 . We defined the $S / P$ ratio as $n / m$.

The whole satellite weighs $140 \mathrm{~kg}$. Since our piezoelectric element is as light as $1.5 \mathrm{~g}$ in weight, all the 50 piezoelectric

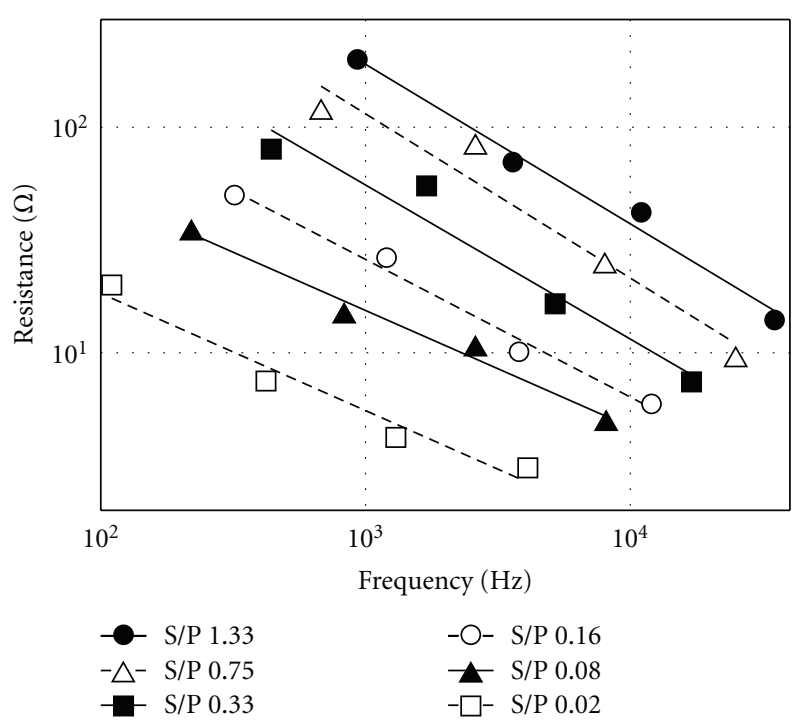

Figure 7: Internal resistance of piezoelectric transducers as a function of measured frequency.

elements have a weight of $75 \mathrm{~g}$. The circuit elements are approximately $50 \mathrm{~g}$. Accordingly, the mass ratio of additional elements (piezoelectric and circuit elements) to the whole satellite is only $0.09 \%$. We need to emphasis that the mass addition of vibration suppression devices is negligibly small.

Figure 6 shows damping capability of our switching method. These experiments carried out a sinusoidal sweep test from $25 \mathrm{~Hz}$ to $100 \mathrm{~Hz}$, while we controlled input force, so that input acceleration remains constant. The horizontal scale is frequency, and the vertical scale is transfer function between two accelerations at top panel and force point. In Figure 6, it is clear that the acceleration transfer function dropped every connection of transducers. The system with $S / P$ ratio 0.5 had the best performance at all, and it is dramatically reduced to as much as the half of original level.

In order to find the cause of the difference between theoretical analysis and experimental results, we focus on internal resistances of our piezoelectric transducers. Figure 7 shows the internal resistance measured at various frequencies by the impedance meter. The horizontal scale is the frequency used at the measurement, and the vertical scale is the measured resistance. In Figure 7, the internal resistances of piezoelectric transducers have a frequency-dependent property. This mechanism and tendency is a similar conclusion that the internal resistance of piezoelectric transducers varies with the number of cycles of an input sinusoidal wave [20].

Figure 8 shows the relation of the voltage ratio after and before voltage inversion, $\gamma$, and connection of piezoelectric transducers. The horizontal scale is a function of series/parallel ratio, and the vertical scale is voltage inversion coefficient. The experimental result agrees with the theoretical analysis result and with the measured value of internal resistance of piezoelectric transducers that is determined at the circuit's electrical natural frequency.

Figure 9 shows the comparison between the experimental result and the theoretical damping capability with the 


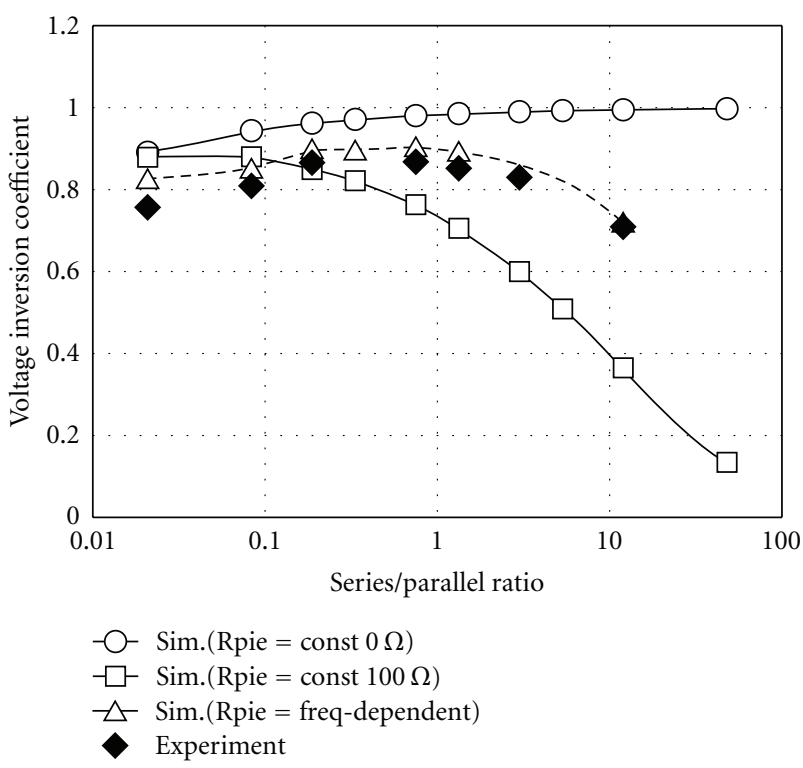

FIgURE 8: Voltage inversion coefficient as a function of series/ parallel ratio.

experimentally measured frequency-dependent resistance in Figure 8. Here, we define a dissipation coefficient as

$$
D_{C} \equiv \frac{1+\gamma}{1-\gamma},
$$

which clearly indicates the essence of damping capability in (10). This can be regarded as the index of theoretical dissipation capacity. On the other hand, experimental dissipation capability is represented by measured acceleration reduction at the top panel. More specifically speaking, this reduction is calculated from the transfer function between two accelerations at the top panel and force point. The theoretical damping capability using frequency-dependent resistance approximately agrees with experimental result. On contrary, the damping capability with constant resistance does not agree with experiment. Therefore, we can draw the conclusion that the connection of multiple piezoelectric transducers needs to be carefully taken into account as well as, their internal resistance. Furthermore, keep in mind that the internal resistance of piezoelectric transducers depends on the frequency measured. This precaution consideration leads to enhancing the damping capability of energyrecycling semiactive vibration suppression.

\section{Conclusions}

This paper presented a comprehensive study on the damping capability of energy-recycling semiactive vibration suppression using multiple piezoelectric transducers. We used the actual satellite "Planet- $\mathrm{A}$ " and 50 sheets of piezoelectric transducers attached to the top panel supporting the astronomical antenna. Firstly, we proved our switching semiactive method worked well on the large-size actual structure in addition to previous small-size experiments, such as simple beams. The acceleration ratio dramatically decreased to

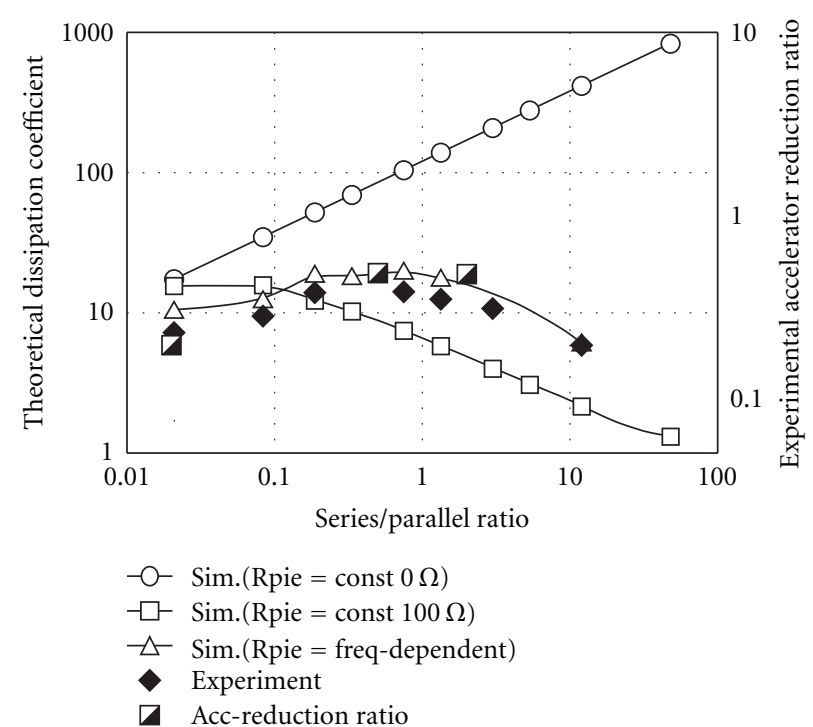

FIGURE 9: Relation of theoretical dissipation coefficient and experimental damping ratio as a function of series/parallel ratio.

0.5 of the original level. This reduction number is quite significant, because we used actual artificial satellite rather than small setups at laboratories.

Secondary, theoretical analyses revealed that a connection of the multiple transducers makes an influence on the damping capability but simultaneously showed interesting discrepancy of damping capability between series and parallel connection.

Thirdly, to find the cause of the discrepancy, we focused on the internal resistance of piezoelectric transducers. We clarified that the discrepancy is caused by the frequencydependent internal resistance of piezoelectric transducers.

One major conclusion is that it is very important to select properly series and parallel connection of multiple piezoelectric transducers. The other major conclusion is that we need to take into account the frequency-dependant internal resistance of piezoelectric transducers. These findings in this paper are very insightful and helpful for developing a system using similar approaches.

\section{Acknowledgment}

This research was supported by a Grant-in-Aid for Scientific Research (B) nos. 1836412 and 19360387, form the Japan Society for Promotion of Science.

\section{References}

[1] J. J. Rodden, H. J. Dougherty, L. F. Rescheke, M. D. Hasha, and L. P. Davis, "Line-of-sight performance improvement with reaction wheel isolation," in Proceedings of the Annual Rocky Mountain, Guidance Control Conference, pp. 71-84, San Diego, Calif, USA, 1989.

[2] M. Ahmadian and A. P. DeGuilio, "Recent advances in the use of piezoceramics for vibration suppression," Shock and Vibration Digest, vol. 33, no. 1, pp. 15-22, 2001. 
[3] C. Niezrecki, D. Brei, S. Balakrishnan, and A. Moskalik, "Piezoelectric actuation: state of the art," Shock and Vibration Digest, vol. 33, no. 4, pp. 269-280, 2001.

[4] M. S. Whorton, J. T. Eldridge, R. C. Ferebee, J. O. Lassiter, and J. W. Redmon, "Damping mechanisms for microgravity vibration isolation," NASA/TM-1998-206953, 1998.

[5] N. W. Hagood and A. Von Flotow, "Damping of structural vibrations with piezoelectric materials and passive electrical networks," Journal of Sound and Vibration, vol. 146, no. 2, pp. 243-268, 1991.

[6] S. Y. Wu, "Piezoelectric shunts with a parallel R-L circuit for structural damping and vibration control," in Smart Structures and Materials 1996: Passive Damping and Isolation, vol. 2720 of Proceedings of SPIE, pp. 259-269, San Diego, Calif, USA, February 1996.

[7] G. A. Lesieutre, "Vibration damping and control using shunted piezoelectric materials," Shock and Vibration Digest, vol. 30, no. 3, pp. 187-195, 1998.

[8] V. Giurgiutiu, "Review of smart-materials actuation solutions for aeroelastic and vibration control," Journal of Intelligent Material Systems and Structures, vol. 11, no. 7, pp. 525-544, 2000.

[9] W. W. Clark, "Vibration control with state-switched piezoelectric materials," Journal of Intelligent Material Systems and Structures, vol. 11, no. 4, pp. 263-271, 2000.

[10] C. Richard, D. Guyomar, D. Audigier, and G. Ching, "Semipassive damping using continuous switching of a piezoelectric device," in Smart Structures and Materials 1999: Passive Damping and Isolation, vol. 3672 of Proceedings of SPIE, pp. 104-111, Newport Beach, Calif, USA, March 1999.

[11] C. Richard, D. Guyomar, D. Audigier, and H. Bassaler, "Enhanced semi passive damping using continuous switching of a piezoelectric device on an inductor," in Smart Structures and Materials 2000: Damping and Isolation, vol. 3989 of Proceedings of SPIE, pp. 288-299, Newport Beach, Calif, USA, March 2000.

[12] L. R. Corr and W. W. Clark, "Comparison of low-frequency piezoelectric switching shunt techniques for structural damping," Smart Materials and Structures, vol. 11, no. 3, pp. 370376, 2002.

[13] J. Onoda, K. Makihara, and K. Minesugi, "Energy-recycling semi-active method for vibration suppression with piezoelectric transducers," AIAA Journal, vol. 41, no. 4, pp. 711-719, 2003.

[14] K. Makihara, J. Onoda, and K. Minesugi, "Novel approach to self-sensing actuation for semi-active vibration suppression," AIAA Journal, vol. 44, no. 7, pp. 1445-1453, 2006.

[15] J. Qiu, H. Ji, and K. Zhu, "Semi-active vibration control using piezoelectric actuators in smart structures," Frontiers of Mechanical Engineering in China, vol. 4, no. 3, pp. 242-251, 2009.

[16] K. Minesugi, J. Onoda, S. Shimose, K. Makihara, and T. Yabu, "Practical application of energy-recycling semi-active vibration suppression method to an actual satellite structural model," in Proceedings of the 58th International Astronautical Congress (IAC '07), vol. 8, pp. 5187-5194, 2007.

[17] B. Jaffe, W. R. Cook Jr., and H. Jaffe, Piezoelectric Ceramics, Academic Press, London, UK, 1971.

[18] M. J. Balas, "Direct velocity feedback control of large space structures," Journal of Guidance, Control, and Dynamics, vol. 2, no. 3, pp. 252-253, 1979.

[19] A. Badel, G. Sebald, D. Guyomar et al., "Piezoelectric vibration control by synchronized switching on adaptive voltage sources: towards wideband semi-active damping," Journal of the Acoustical Society of America, vol. 119, no. 5, pp. 28152825, 2006.

[20] K. Makihara, J. Onoda, and K. Minesugi, "Behavior of piezoelectric transducer on energy-recycling semiactive vibration suppression," AIAA Journal, vol. 44, no. 2, pp. 411-413, 2006. 

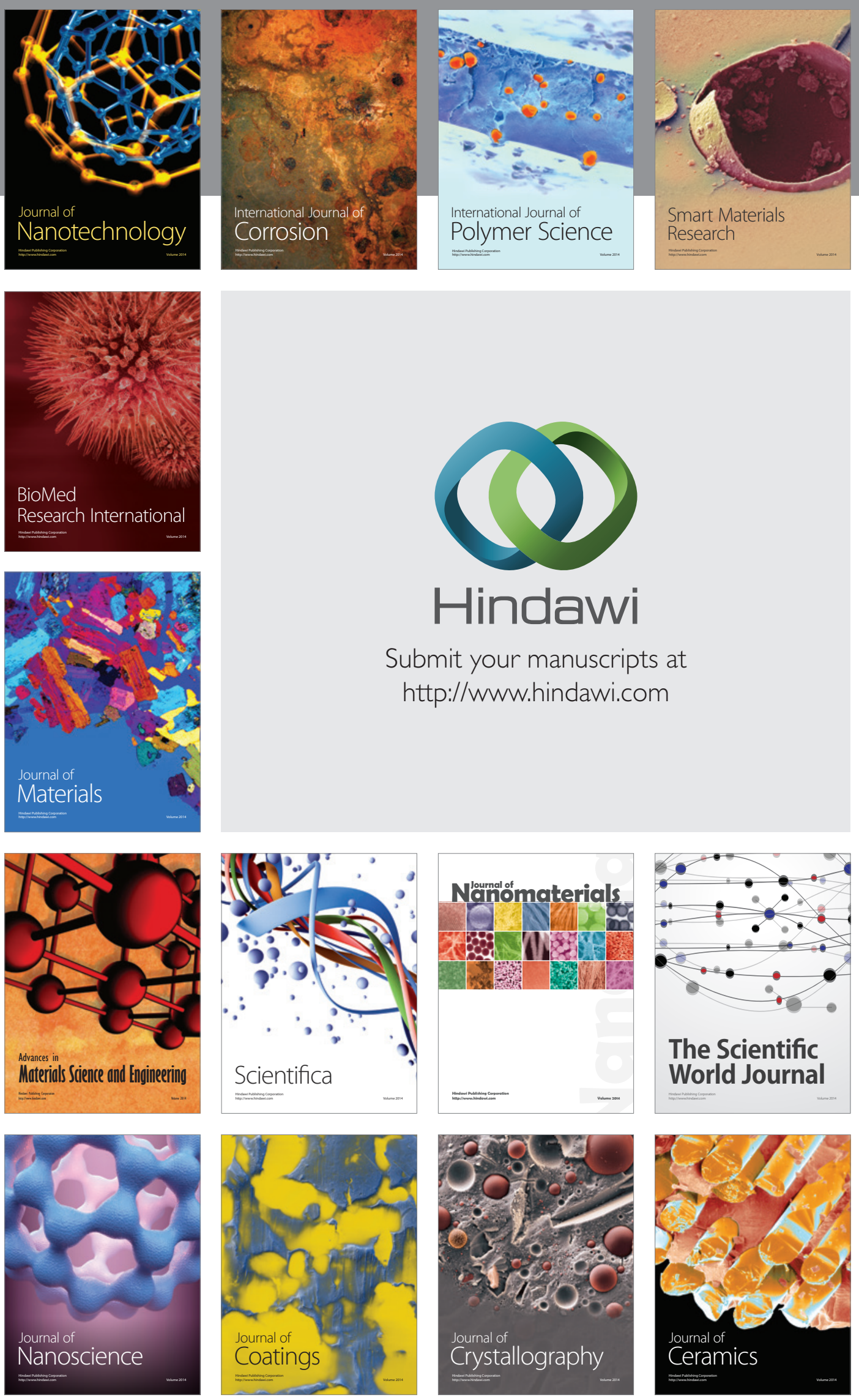

The Scientific World Journal

Submit your manuscripts at

http://www.hindawi.com

\section{World Journal}

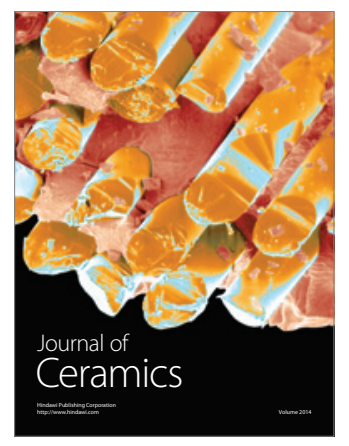

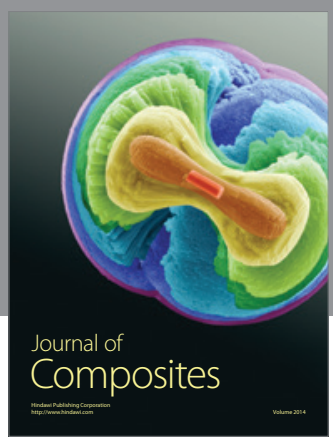
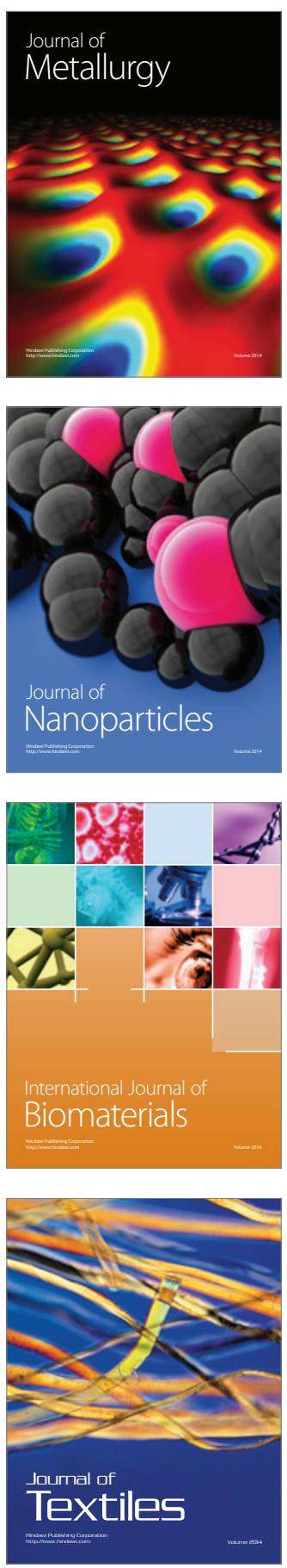